\title{
The X17.2 flare occurred in NOAA 10486: an example of filament destabilization caused by a domino effect
}

\author{
F. Zuccarello ${ }^{1}$, P. Romano ${ }^{2}$, F. Farnik ${ }^{3}$, M. Karlicky ${ }^{3}$, L. Contarino ${ }^{2}$, V. Battiato ${ }^{2}$, S. L. Guglielmino ${ }^{1}$, \\ M. Comparato ${ }^{1}$, and I. Ugarte-Urra ${ }^{4}$
}

1 Dipartimento di Fisica e Astronomia - Sezione Astrofisica, Università di Catania, via S. Sofia 78, 95123 Catania, Italy e-mail: fzu@oact.inaf.it

2 INAF - Osservatorio Astrofisico di Catania, via S. Sofia 78, 95123 Catania, Italy

3 Astronomical Institute, Academy of Sciences, 25165 Ondřejov, Czech Republic

${ }^{4}$ Space Science Division, Naval Research Laboratory, Washington, DC 20375, USA

Received 1 April 2008 / Accepted 17 October 2008

ABSTRACT

\begin{abstract}
Context. It is now possible to distinguish between two main models describing the mechanisms responsible for eruptive flares : the standard model, which assumes that most of the energy is released, by magnetic reconnection, in the region hosting the core of a sheared magnetic field, and the breakout model, which assumes reconnection occurs at first in a magnetic arcade overlaying the eruptive features.

Aims. We analyze the phenomena observed in NOAA 10486 before and during an X17.2 flare that occurred on 2003 October 28, to study the relationship between the pre-flare and flare phases and determine which model is the most suitable for interpreting this event.

Methods. We performed an analysis of multiwavelength data set available for the event using radio data $(0.8-4.5 \mathrm{GHz})$, images in the visible range (WL and $\mathrm{H}_{\alpha}$ ), EUV images (1600 and $195 \AA$ ), and X-ray data, as well as MDI longitudinal magnetograms. We determined the temporal sequence of events occurring before and during the X17.2 flare and the magnetic field configuration in the linear force-free field approximation.

Results. The active region was characterized by a multiple arcade configuration and the X17.2 flare was preceded, by $\sim 2 \mathrm{~h}$, by the partial eruption of one filament. This eruption caused reconnection at null points located in the low atmosphere and a decrease in magnetic tension in the coronal field lines overlaying other filaments present in the active region. As a consequence, these filaments were destabilized and the X17.2 flare occurred.

Conclusions. The phenomena observed in NOAA 10486 before and during the X17.2 flare cannot be explained by a simple scenario such as the standard or breakout model, but instead in terms of a so-called domino effect, involving a sequence of destabilizing processes that triggered the flare.
\end{abstract}

Key words. Sun: activity - Sun: flares - Sun: magnetic fields - Sun: filaments

\section{Introduction}

The analysis of data acquired by space-born missions, such as the YOHKOH, SOHO, and TRACE satellites, had helped us in understanding the physics of flares and CMEs and formulating theoretical models to explain the physical mechanisms involved in these events. These models claim that some magnetic field destabilizing elements, such as the emergence of new magnetic flux or photospheric flows, produce shearing of the magnetic field and an increase in magnetic energy and loss of equilibrium. As a consequence, the magnetic field releases the surplus of energy by means of magnetic reconnection, in such a manner as to rearrange itself in a new, less energetic, equilibrium configuration.

Theoretical models can have different initial magnetic configurations and physical mechanisms that trigger the eruption. The standard 2D model, developed by Carmichael (1964), Sturrock (1966), Hirayama (1974), Kopp \& Pneuman (1976), and Priest \& Forbes (2000), assumes an initial bipolar magnetic configuration, involving therefore only one magnetic arcade. In this model, most of the energy is released in the region where the core of the sheared magnetic field is located, and the eruption is the result of the reconnection between flux elements internal to the core of the field itself.

A different source of magnetic energy for the eruption of flares and CMEs might be found in the surrounding field, as proposed by the magnetic breakout model (Antiochos et al. 1999). This model can be applied only in multipolar configurations, where the core of the sheared magnetic field is contained by the magnetic field lines of an overlaying arcade. On the basis of this model, the eruption causing the flare or CME may happen only after a break in the overlaying field lines, related to a process of magnetic reconnection initiating in a neutral point located outside the region hosting the core of the sheared magnetic field.

We investigated several phenomena that occurred before and during an X17.2 eruptive flare, followed by an Earth-directed CME, observed in NOAA 10486 on 2003 October 28, to determine which of the cited models, is the most suitable for interpreting the sequence of phenomena observed.

The flare studied occurred in a region of complex magnetic field topology, that had already been investigated by several authors (see e.g. Pick et al. 2005, Hurford et al. 2006, Mandrini et al. 2006, Schmieder et al. 2006a, Su et al. 2006, 


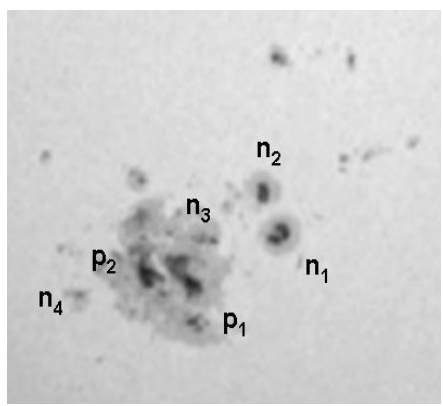

(a)

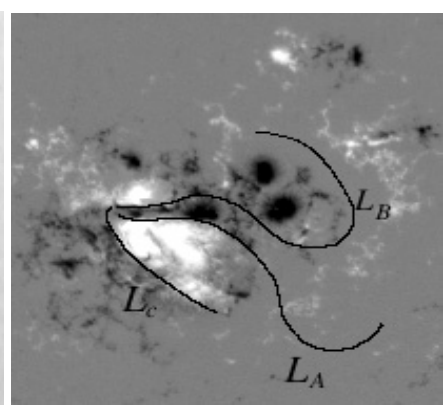

(b)

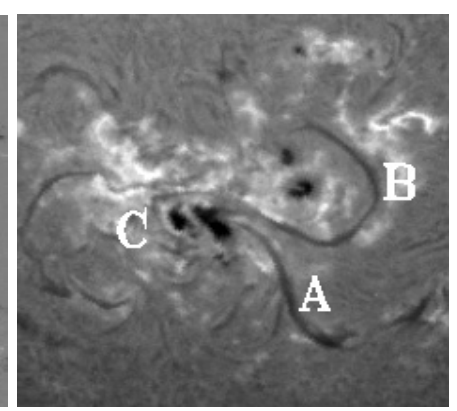

(c)

Fig. 1. a) Photospheric image of NOAA 10486 acquired at INAF-OACt on 28 October at 8:29 UT; $n_{1}, n_{2}, n_{3}$ and $n_{4}$ indicate the sunspots of negative polarity, while $p_{1}$ and $p_{2}$ are the main positive polarity spots; b) MDI magnetogram showing the magnetic configuration of NOAA 10486 at 08:00 UT; $L_{A}, L_{B}$ and $L_{C}$ indicate the main neutral lines; c) $\mathrm{H}_{\alpha}$ image acquired at INAF-OACt at 7:37 UT. The labels $A, B$, and $C$ indicate three filaments involved in the flaring activity of NOAA 10486 that are located along the neutral lines $L_{A}, L_{B}$, and $L_{C}$, respectively. The field of view of these images is $\sim 335 \times 335 \mathrm{Mm}^{2}$. In these and in the following images, if not specified otherwise, North is at the top, West at the right.

Li et al. 2007, and Trottet et al. 2008), although there is no common interpretation of the sequence of events characterizing its evolution.

\section{Data description}

To study the morphology and evolution of NOAA 10486, we used radio data $(0.8-4.5 \mathrm{GHz})$ acquired by the Ondřejov radiospectrograph, WL and $\mathrm{H}_{\alpha}$ images acquired at INAF-Catania Astrophysical Observatory (INAF-OACt), 195 and $1600 \AA$ images taken by TRACE and data acquired by RHESSI in the 15-100 keV band. We also used full-disk line-of-sight magnetograms acquired by $\mathrm{MDI} / \mathrm{SOHO}$.

Ondřejov radio data have a time resolution of $0.1 \mathrm{~s}$ for the $0.8-4.5 \mathrm{GHz}$ radiospectrographic data, and a time resolution of $0.01 \mathrm{~s}$ for single frequency recording at $3 \mathrm{GHz}$ (Jiřička et al. 1993).

INAF-OACt images were recorded by a $1200 \times 1360 \mathrm{CCD}$ detector with a spatial resolution of 2 arcsec and a dynamic range of 16 bits. In standard patrol mode, the $\mathrm{WL}$ and $\mathrm{H}_{\alpha}$ images are acquired with a time cadence of $1 \mathrm{~h}$ and $15 \mathrm{mn}$, respectively, while the $\mathrm{H}_{\alpha}$ images taken on 28 October between 7:30 UT and 8:10 UT have an average time cadence of about $2 \mathrm{mn}$ (time cadence adopted during flares and erupting filaments).

TRACE images were taken in full resolution mode, i.e. $768 \times$ 768 pixel, with a pixel size of 0.5 arcsec and a field of view of $6.4 \times 6.4$ arcmin; the time cadence was $\sim 30 \mathrm{~s}$.

The RHESSI spacecraft provides imaging spectroscopy at high time resolution, and angular resolution of 2.26 arcsec, in the energy range $3 \mathrm{keV}-17 \mathrm{MeV}$ (Lin et al. 2002). RHESSI data have been analyzed using the standard SolarSoft procedure by the CLEAN method.

The MDI magnetograms that we used, characterized by a spatial resolution of $\sim 2$ arcsec, were taken on 28 October between 07:53 and 12:00 UT.

\section{NOAA 10486 global morphology and evolution}

NOAA 10486 appeared at the East limb on 2003 October 22, with an average latitude of $18 \mathrm{~S}$, and disappeared at the West limb on 5 November.

The configuration of NOAA 10486 is shown in Fig. 1: by comparing the WL image (Fig. 1a) and the MDI magnetogram (Fig. 1b), it is possible to infer that, in the western side of the AR, two sunspots of negative polarity and roughly circular shape are present $\left(n_{1}\right.$ and $\left.n_{2}\right)$. In the central part, there is a wide region containing two large sunspots $\left(p_{1}\right.$ and $\left.p_{2}\right)$ and several smaller sunspots of positive polarity inside the same penumbra, which is divided into two parts by a relatively thin region of negative polarity. Along this thin region it is possible to notice one small sunspot of negative polarity $\left(n_{3}\right)$, apparently inside the same penumbra containing the positive sunspots: due to these characteristics, this area can be considered to be a $\delta$ spot. In the northern and eastern parts of the AR, other relatively small sunspots are present, and we label $n_{4}$ the eastern negative spot.

In the magnetogram shown in Fig. $1 \mathrm{~b}$, three main neutral lines, indicated by $L_{A}, L_{B}$, and $L_{C}$, have been drawn. We notice that these neutral lines appear to have a contact point in the region of negative intrusion and that, in particular, $L_{A}$ and $L_{B}$ are very close to each other in this region. Previous analysis of this active region demonstrated that this magnetic configuration was the result of complex evolution, characterized by two successive events of flux emergence (Schmieder et al. 2006a).

The chromospheric configuration of NOAA 10486 is shown in the $\mathrm{H}_{\alpha}$ image of Fig. 1c, in which we can observe several filaments in and around the active region. We indicated three of them, located along the neutral lines $L_{A}, L_{B}$ and $L_{C}$, by the letters A-C respectively. In particular, the filament A is characterized by negative magnetic helicity, which we can infer from its reverse S-shape (Rust 2003) and its chirality (Martin 2003). As described in the following, an X17.2 flare was preceded, by $\sim 2 \mathrm{~h}$, by the partial eruption of this filament, involving the two magnetic arcades that contain filaments B and C (hereafter arcades B and $\mathrm{C}$, respectively).

\section{The event evolution}

The solar X-ray flux recorded by GOES-11 (see Fig. 2) indicates that on 28 October an X17.2 flare occurred, beginning at 9:51 UT and reaching the peak at 11:10 UT. Imaging instruments indicated that the flare occurred in NOAA 10486 and that it was associated with an Earth-directed CME that left the Sun at approximately 11:04 UT (Pick et al. 2005).

\subsection{Filament $A$ activation and partial eruption (7:54-8:11 UT)}

At 7:53 UT, i.e. about $2 \mathrm{~h}$ before the beginning of the X17.2 flare, the eruption of filament A was observed in the $\mathrm{H} \alpha$ line and at $1600 \AA$, and became observable a few minutes later at 


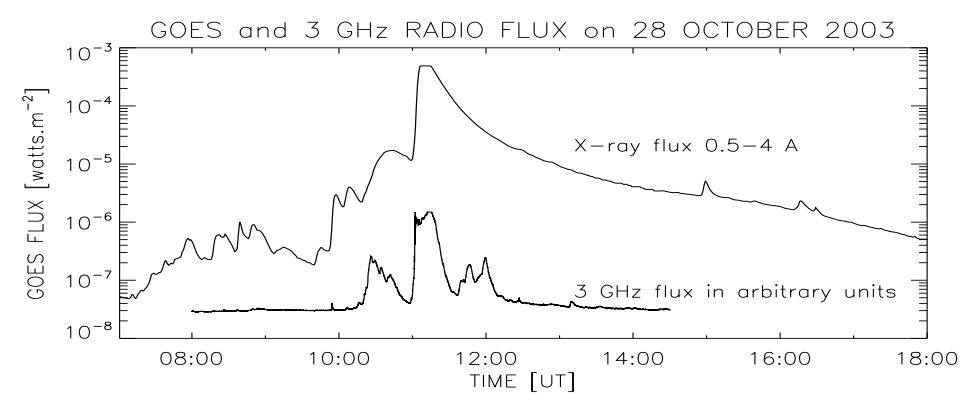

Fig. 2. GOES-11 recorded X-ray flux in the $0.5-4 \AA$ band on 28 October 2003 together with the $3 \mathrm{GHz}$ radio flux recorded by the Ondřejov radiospectrograph. The graph shows the occurrence of an X17.2 flare, with a peak of intensity at 11:10 UT.

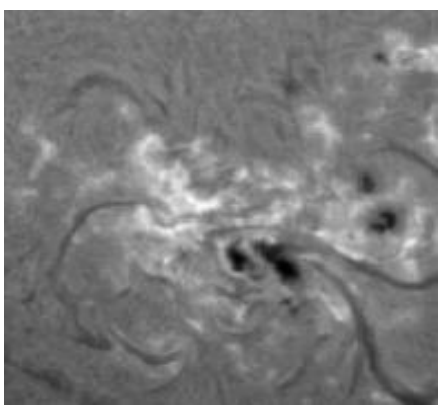

(a)

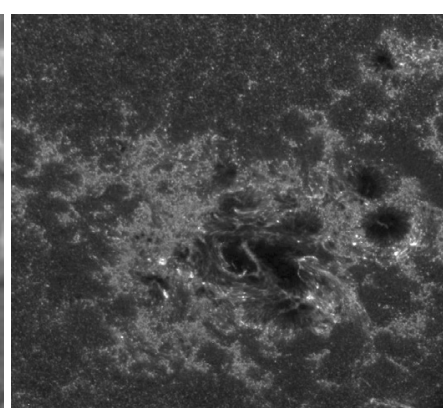

(b)

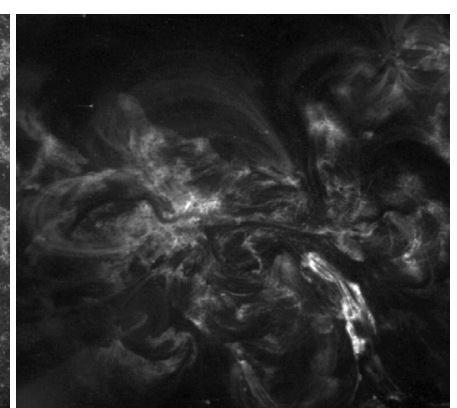

(c)

Fig. 3. Sequence of images showing the eruption of filament A: a) $\mathrm{H} \alpha$ image acquired at INAF-OACt at the beginning of filament A eruption (7:53 UT); b) $1600 \AA$ TRACE image showing the beginning of the filament eruption at a higher atmospheric level (7:53 UT); c) $195 \AA$ TRACE image showing the twisted configuration of filament A during its eruption (7:56 UT). The field of view of these images is $\sim 280 \times 280 \mathrm{Mm}^{2}$ $(\sim 384 \times 384$ arcsec $)$.

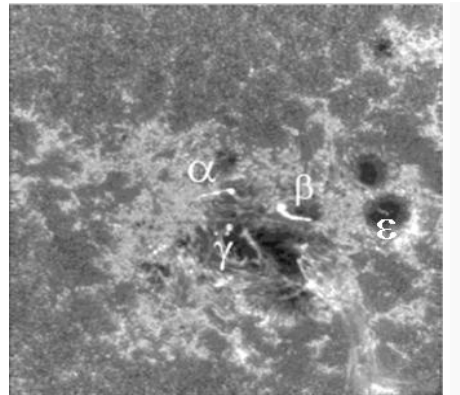

(a)

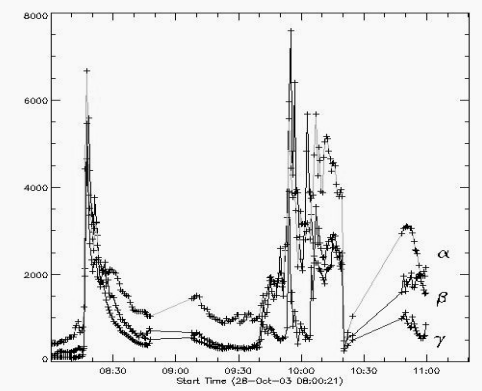

(b)

Fig. 4. a) $1600 \AA$ image showing the locations of the regions $\alpha, \beta, \gamma$, and $\epsilon$ (see text); the field of view of this image is $\sim 280 \times 280 \mathrm{Mm}^{2}$ $(\sim 384 \times 384 \mathrm{arcsec})$; b) light curves showing the brightness variations during the time interval 08:00:21-10:59:46 UT in the regions $\alpha, \beta$, and $\gamma$ (compare with Fig. 7). During the time intervals 8:47-9:07 and 10:24-10:47 UT, there were gaps in the observations.

$195 \AA$ (see Fig. 3). At this wavelength, during the main phase of this eruption, we note a left helical flux-rope structure (Fig. 3c), which suggests that filament $\mathrm{A}$ has a twisted configuration and provides further evidence of its negative helicity (Chae 2001).

The eruption of the filament was observed until 7:59 UT. At later times, the filament developed a bifurcate shape in the $\mathrm{H} \alpha$ images, while in TRACE images the brightening associated with the filament eruption decreased until 8:11 UT, when it was no longer visible.

\subsection{Filament $B$ brightening and activation (8:11-10:16 UT)}

The eruption of filament A appears to have caused destabilization in the active region and eventually the X17.2 flare. The $1600 \AA$ and $195 \AA$ images acquired by TRACE detected in fact an intense pre-flare phase, starting with brightenings appearing $\sim 16 \mathrm{Mm}$ from the location where the northern footpoint of filament A was located, around filament B, and in the south-west part of sunspot $n_{1}$ (see Fig. 4a, where they are indicated by the letters $\alpha, \beta, \gamma$, and $\epsilon$ ).

Regions $\alpha, \beta$, and $\gamma$ : at $1600 \AA$, these bright knots, first observed at 8:16 UT, exhibit a continuous brightness increase. Appearing to be the contact region between filaments A and B and located on the same neutral line as filament $\mathrm{B}$, the bright knot $\beta$, since 8:18 UT, increases its dimensions and becomes visible as a bright filamentary structure. This feature is spatially coincident with filament $\mathrm{B}$, and at 8:27 UT, reaches a length of $\sim 84 \mathrm{Mm}$. After a period of dimming, the regions $\alpha, \beta$, and $\gamma$ increase their brightness again, and, from 8:40 UT, $\alpha$ and $\beta$ appear to join and to increase in length along the same neutral line forming a bright structure of length $\sim 100 \mathrm{Mm}(8: 43 \mathrm{UT})$ and spatially coincident with filament B. 


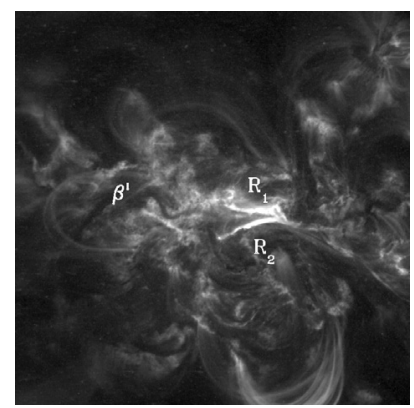

(a)

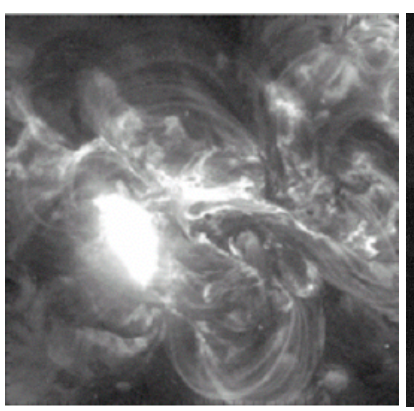

(b)

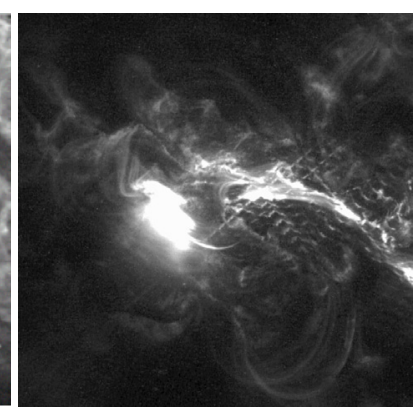

(c)

Fig. 5. Flare evolution at $195 \AA$ : a) at 10:04 UT, in the center of the active region, a bright feature $\left(\beta^{\prime}\right)$ and two bright ribbons $\left(R_{1}\right.$ and $\left.R_{2}\right)$ are visible; b) at 10:47 UT, the C arcade is the brightest site in the AR; c) at 11:01 UT, the C arcade is less bright, while the filament B appears very bright. The field of view of these images is $\sim 280 \times 280 \mathrm{Mm}^{2}(\sim 384 \times 384 \mathrm{arcsec})$.

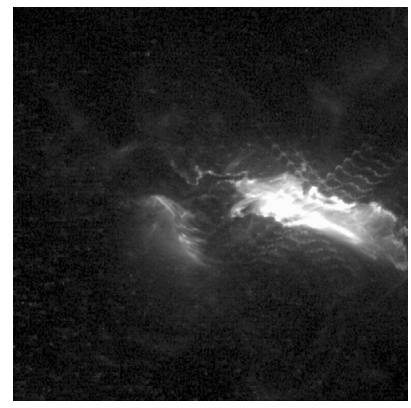

(a)

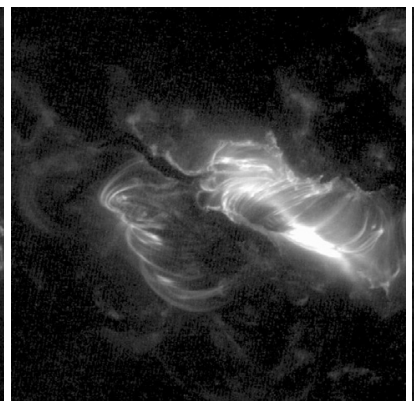

(b)

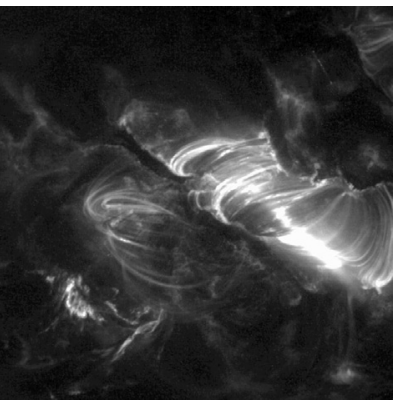

(c)

Fig. 6. Images showing the flare evolution at $195 \AA$ A: a) 11:06 UT: the ribbons of the B arcade are clearly visible; b) at 11:48 UT, the loops of the $\mathrm{B}$ arcade are larger, while the $\mathrm{C}$ arcade decreases in brightness; c) 12:27 UT; both C and B arcades are evident. The field of view of these images is $\sim 280 \times 280 \mathrm{Mm}^{2}(\sim 384 \times 384 \mathrm{arcsec})$.

There was then a gap in the observations and, at 9:08 UT, the $\alpha, \beta$, and $\gamma$ knots were decreasing in brightness. They appeared again at 9:41 UT. At 9:54 UT, $\beta$ extended along the filament B of length $\sim 100 \mathrm{Mm}$ and $\gamma$ appears again. At later times, filament B decreases its brightness until disappearing, but, at 10:16 UT, it is bright again and has a length of $\sim 150 \mathrm{Mm}$.

In synthesis, the lengthening and brightening of this region is observed 4 times, and each time with increasing length.

In Fig. $4 \mathrm{~b}$, the brightness temporal variation of the aforementioned regions is shown: these curves indicate that there are three main peaks (at approximately 8:20, 09:55, and 10:15 UT).

Region $\epsilon$ : since 8:16 UT, a bright knot is observed in $1600 \AA$ images in region $\epsilon$. The luminosity increases until 8:27 UT, when it reaches a maximum and the region $\epsilon$ has the shape of a reversed Y. At a later time, the $\epsilon$ luminosity decreases.

\subsection{Brightening of $B$ and $C$ arcades (10:00-12:32 UT)}

After a gap in the $195 \AA$ Abservations, at 10:04 UT, two bright filamentary structures, labeled $R_{1}$ and $R_{2}$, and located on the north side of sunspot $p_{1}$, were observed; at the same time, a bright feature, called $\beta^{\prime}$, was observed close to the northern footpoints of the $\mathrm{C}$ arcade (see Fig. 5a).

The bright region $\beta$ ' then increased in brightness and, at 10:47 UT, the entire $C$ arcade reached its maximum brightness (see Fig. 5b). At 11:01 UT, filament B was very bright and $5 \mathrm{mn}$ later two bright ribbons, approximately parallel to filament B, were detected (see Figs. 5c and 6a). At 11:48 UT, the B arcade was clearly visible (see Fig. 6b). At 12:27 UT, it was possible to observe the bright loops of both the $\mathrm{C}$ and $\mathrm{B}$ arcades (see Fig. 6c).
Using TRACE $195 \AA$ Amages, we measured the separation velocity of the ribbons observed during the eruption of filament B. The velocities were at their highest values at the flare peak $\left(\sim 100 \mathrm{~km} \mathrm{~s}^{-1}\right)$, and decreased immediately after. We recall that the separation motion of flare ribbons provides a measure of the rate of magnetic reconnection in the corona ( $\mathrm{Li}$ et al. 2007), which implies that this process occurred rapidly at the flare peak and later on slowed down.

Using an IDL procedure based on a method developed by Loughhead et al. (1983), we calculated the azimuthal and inclination angles of the post-flare loops belonging to the $\mathrm{B}$ arcade (see Contarino et al. (2006) for a description of the procedure). The results indicate that both the azimuthal and inclination angles decreased during the main phase (from 40 to 25 degrees and from 21 to 17 degrees, respectively), which implies that the reconnected loops exhibit with time a less stressed configuration and more vertical position with respect to the solar surface.

\subsection{Analysis of radio data}

A global overview of the $3 \mathrm{GHz}$ radio-flux variations in the time interval 08:00-14:30 UT and the GOES-11 light curve is presented in Fig. 2. According to the radio burst activity, the complete time interval can be divided into three parts (Fig. 7): a) Preflare activity (08:00-10:15 UT), b) first part of the flare (10:15-10:58 UT), and c) second part of the flare (10:58-12:30 UT).

To illustrate the preflare activity, we have no observations before 08:00 UT, but the $3 \mathrm{GHz}$ radio flux record (Fig. 7, upper panel) suggests that the preflare activity started before this time. At 08:15-08:54 UT, this activity then increased. After its 

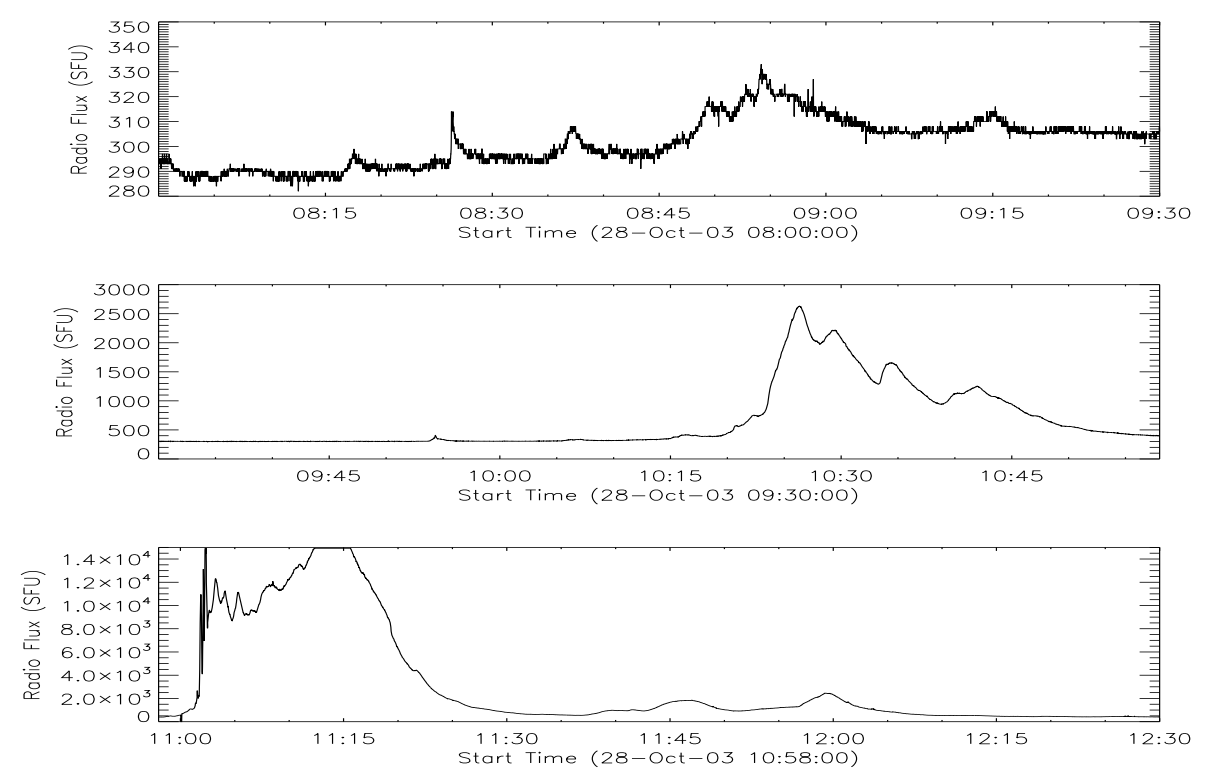

Fig. 7. The $3 \mathrm{GHz}$ radio fluxes observed during the October 28, 2003 event, in the time intervals 08:00-09:30 UT upper panel), 09:30-0:58 UT (middle panel), and 10:58-12:30 UT (bottom panel) by the Ondřejov radiospectrometer.

Table 1. Radio-burst activity in the $40-800 \mathrm{MHz}$ frequency range at 07:45-11:00 UT observed by the Potsdam-Tremsdorf radiospectrograph (Courtesy of Aurass).

\begin{tabular}{ccc}
\hline \hline $\begin{array}{c}\text { Time } \\
\text { interval (UT) }\end{array}$ & $\begin{array}{c}\text { Frequency } \\
\text { interval (MHz) }\end{array}$ & Burst types \\
\hline 10:42-10:48 & $400-800$ & $\begin{array}{c}\text { group weak type III bursts } \\
\text { type III burst noise storm } \\
\text { 07:45-10:15 }\end{array}$ negatively drifting chains \\
& $200-400$ & $\begin{array}{c}\text { group of strong type III bursts } \\
\text { type III burst }\end{array}$ \\
10:15-11:00 & $200-500$ & group of type III bursts \\
$07: 45-07: 48$ & $50-75$ & group of type III bursts \\
08:53-08:56 & $40-80$ & \\
10:00-11:00 & $40-85$ & \\
\hline
\end{tabular}

maximum, the activity decreased and a new pulse of activity was recorded at 09:53:50-09:55:30 UT, where a group of highfrequency type III bursts were observed in the $2.0-4.5 \mathrm{GHz}$ frequency range (Fig. 8a). These type III bursts indicate the presence of beams of accelerated electrons at densities of about $10^{11} \mathrm{~cm}^{-3}$. Thus, similar densities can be expected at locations where these electrons are accelerated, probably by a magnetic reconnection process.

The first part of the flare is evident at $3 \mathrm{GHz}$ (in the middle panel of Fig. 7). This event started at about 10:15 UT, reaching its maximum (2600 SFU) at 10:26 UT, and ending at 10:58 UT, when the second part of the flare started. It is interesting that the $3 \mathrm{GHz}$ radio flux oscillates quasi-periodically with characteristic periods of 3-5 $\mathrm{mn}$. These oscillations were also observed in the $0.8-4.5 \mathrm{GHz}$ spectrum, where broadband pulses and continua were detected. The high-frequency bursts indicate reconnection processes at densities $10^{10}-10^{11} \mathrm{~cm}^{-3}$.

The entire time interval 07:45-11:00 UT for the $40-800 \mathrm{MHz}$ range was characterized by a noise storm of type III bursts, especially in the $200-400 \mathrm{MHz}$ range (see Table 1). This burst activity indicated that at altitudes in the corona (in particular at about $90000 \mathrm{~km}$ above the photosphere, considering the 4 times Newkirk model and plasma emission at the fundamental frequency) electron beams were accelerated in reconnection processes during the aforementioned time interval.
The second part of the flare, as shown in Fig. 7 (bottom panel), started at 10:58 UT. First, the radio flux at $3 \mathrm{GHz}$ increased exponentially until 11:02 UT, and then, after the quasiperiodic flux oscillations (11:02-11:13 UT) of periods in the range 1-3 $\mathrm{mn}$, the flux continued to increase to an incredibly high saturation level. After 11:16 UT, the $3 \mathrm{GHz}$ radio flux decreased until the flare ended, except for two post-flare activity peaks at 11:46 and 11:59 UT.

For more details about the radio emission in the $40-800 \mathrm{MHz}$ frequency range at the second part of the flare (starting at 10:58 UT), we refer the reader to Aurass et al. (2006, 2007) and Rausche et al. (2007).

The $0.8-4.5 \mathrm{GHz}$ radio spectrum at these times had an exceptionally strong continuum, especially around the flare maximum. On the other hand, shortly before and after this strong continuum, the event was rich in fine structures, such as drifting pulsation structures (DPS), broadband pulses, narrow-band pulsations, and at the ending flare phase also zebras, and fibers. The most significant fine structures are the drifting pulsating structures at the beginning of this enormous flare (see Fig. 8b). In the spectrum (Fig. 8b), there are two parallel, drifting, pulsating structures, one in the range $0.8-1.3 \mathrm{GHz}$, and the second at higher frequencies of $1.2-1.8 \mathrm{GHz}$. Their frequency drift was about 1.7 $\mathrm{MHz} \mathrm{s}^{-1}$. At 11:01:19-11:01:22 UT, these DPSs were accompanied by a reverse drift burst in the $1.6-2.0 \mathrm{GHz}$ range, which is indicative of electron-beam bombarding deep inside the chromospheric layers.

As proposed by Kliem et al. (2000) and Karlický (2004), these drifting pulsating structures are generated by the plasmaemission mechanism of superthermal electrons trapped inside the plasmoids which were formed in the reconnecting current sheet. Assuming that the radio emission is produced at the plasma frequency of the background plasma, the density of these two plasmoids can be estimated to be $1.5 \times 10^{10}$ and $2.8 \times 10^{10} \mathrm{~cm}^{-3}$, respectively. The plasmoids move upwards in the flare atmosphere as indicated by the negative frequency drift of the DPSs. The process is called plasmoid ejection, which was described by Ohyama \& Shibata (1998). 


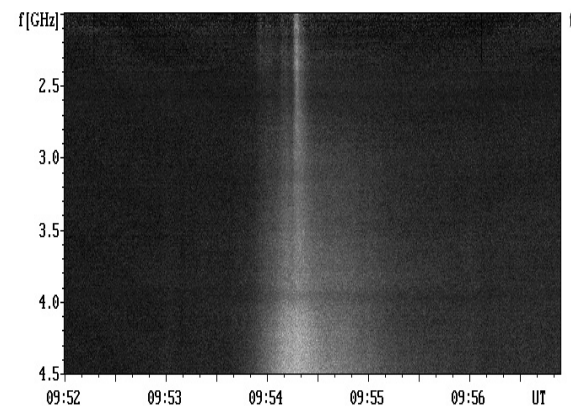

(a)

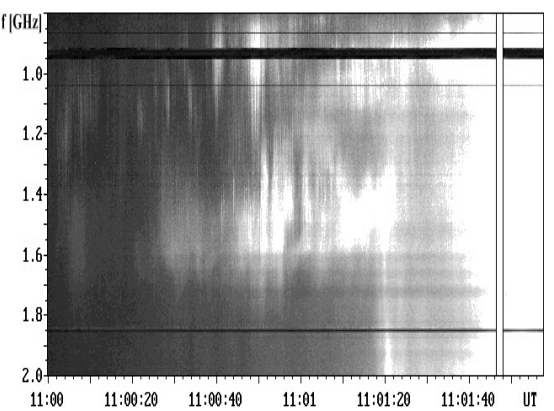

(b)

Fig. 8. a) The 2.0-4.5 GHz radio spectrum observed at 09:52-09:54 UT showing a group of the high-frequency type III bursts; b) the 0.8-2.0 GHz radio spectrum observed at 11:00:00-11:01:50 UT showing two parallel negatively drifting pulsating structures (DPSs) accompanied by the reverse drift burst at 11:01:19-11:01:22 UT in the 1.6-2.0 GHz range.

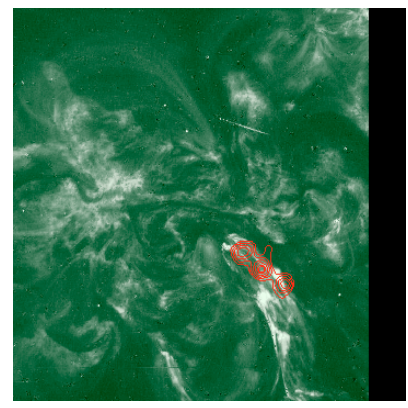

(a)

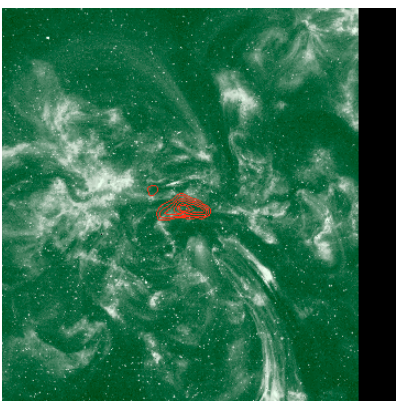

(b)

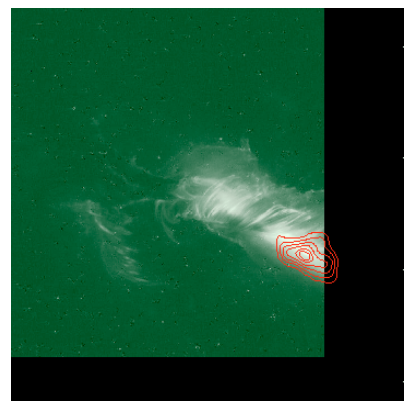

(c)

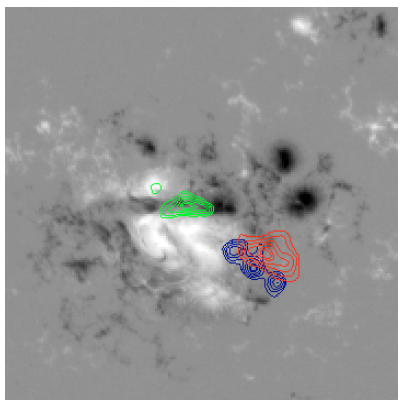

(d)

Fig. 9. a) Overplot of the RHESSI image (red contours) over the TRACE image (195 ^) taken at 07:58:40 UT; the RHESSI image is integrated for $28 \mathrm{~s}$ around 07:55:42 UT in the 15-50 keV energy band, contour levels at 50,60, 70, 80, 90, and 95\% of maximum intensity; b) TRACE image (195 ̊) taken at 08:08:57 UT, RHESSI image integrated for $60 \mathrm{~s}$ around 08:16:30 UT in the 15-50 keV energy band; c) TRACE image (195 $\AA$ ) taken at 11:21:38 UT, RHESSI image is integrated for $16 \mathrm{~s}$ around 11:22:08 UT in the 25-100 keV energy band. d) Overplot of the RHESSI hard X-ray sources over the MDI magnetogram acquired at 07:53:03 UT. The position of the X-ray source at 11:22:08 UT was corrected on solar rotation and is correct relative to the background magnetic field. The source indicated by blue isocontours was visible at 07:55:42 UT, the source indicated by green isocontours at 08:16:30 UT, and the source indicated by red isocontours during the main phase, i.e., at 11:22:08 UT.

\subsection{X-ray data description and analysis}

$\mathrm{X}$-ray data used in this analysis were acquired by the GOES and RHESSI satellites. GOES data monitored soft X-ray emission during the entire event (see Fig. 2), detecting an increase in X-ray flux at 0.5-4 $\AA$ band, that started during the eruption of filament A. At a later time, the X-ray flux exhibited a spiky behavior, which was similar to that shown by the light curves derived from radio data at $3 \mathrm{GHz}$ and by TRACE $1600 \AA$ images (see Fig. 4b).

RHESSI observations were affected by night and radiation belt periods, therefore we could use only three short periods between the start and main phase of the event in the hard X-ray imaging: 07:45-08:16, 09:40-09:50, and 11:05-11:30 UT.

Reconstructed RHESSI images acquired during these periods were superimposed on the TRACE images. The TRACE image coordinates were corrected by using SOHO/EIT images as the reference. Unfortunately, the EIT data were saturated during the main phase of the flare and therefore the precision of the co-alignment can be slightly worse during the main phase.

Figure 9 (a) shows that, at 07:55:42 UT, the hard X-ray isocontours correlate spatially with the part of the erupting filament A closer to filament $\mathrm{B}$, which indicates that most of the energetic particles were accelerated in this site.

At 08:16:30 UT, the source of hard X-ray is located above the intrusion of negative polarity (see Fig. 9b), while, at 11:22:08 UT, it corresponds to the footpoints of the B arcade seen in the TRACE $195 \AA$ images (see Fig. 9c).
To illustrate how the position of the hard X-ray source develops with time, we present, in Fig. 9d, the RHESSI hard X-ray sources superimposed on the MDI magnetogram acquired at 07:53:03 UT. Two X-ray sources correlate well in time with the magnetogram, while the position of the third source (at 11:22:08 UT) was corrected for solar rotation and its relative position with respect to magnetic field is therefore also correct.

During the eruption of filament A, we can see that the hard $\mathrm{X}$-ray source coincides with its location. At a later time, the source corresponds to the negative polarity intrusion and, finally, during the flare main phase, its spatial position correlates well with that of the B arcade and in part with the X-ray isocontours observed during the eruption of filament A.

\section{Analysis of the magnetic configuration}

To investigate the configuration of the magnetic field of NOAA 10486 at coronal levels, we used an extrapolation code based on a method introduced by Alissandrakis (1981). The model assumes that the magnetic field is force-free both in the corona and at lower levels, and vanishes at infinity. Using the assumption of a constant $\alpha$ force-free field, the equation:

$\nabla \times \boldsymbol{B}=\alpha \boldsymbol{B}$

can be numerically solved using Fourier transforms. The code assumes as its input the value of the longitudinal magnetic field component at the boundary (i.e. the photosphere) provided by 


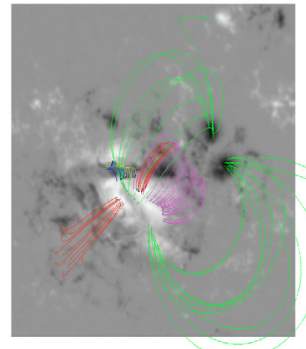

(a)

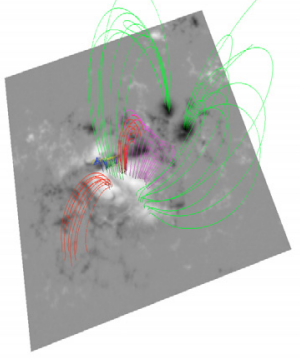

(b)

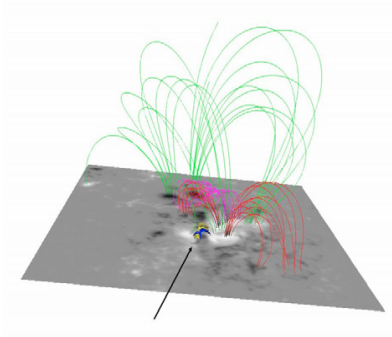

(c)

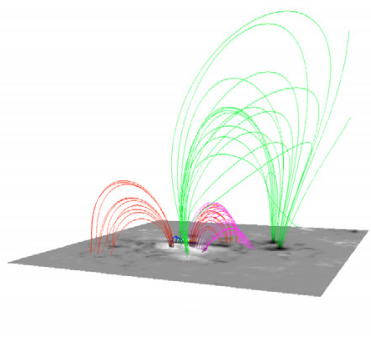

(d)

Fig. 10. a) Overplot of the magnetic field lines inferred from the force-free field extrapolation on the MDI magnetogram acquired at 07:53 UT; b) the same field of view shown in a), but rotated $\sim 30^{\circ}$ counterclockwise; c) the same field of view shown in a), but rotated $\sim 90^{\circ}$ counterclockwise and with an inclination of $\sim 60^{\circ}$; $\mathbf{d}$ ) the same field of view shown in a), but with an inclination of $\sim 90^{\circ}$.

MDI magnetograms. Using this method, it is possible to reconstruct the 3-D magnetic field above the photosphere, obtaining as output three 3-D arrays with the values of each component of the magnetic field in 3-D space.

In particular, the magnetic field extrapolation was performed on magnetograms centered on the active region such that the maximum magnetic flux imbalance was less than $20 \%$. To minimize the oscillatory behavior of the Fourier solutions, we placed the original arrays of data for the boundary side in larger arrays of double the size of the original arrays, before applying the extrapolation procedure. The size of the integration boxes $(\sim 545 \times 545 \mathrm{Mm})$ infers a resonant value of the $\alpha$ force free parameter of $1.50 \times 10^{-2} \mathrm{Mm}^{-1}$; therefore, we assumed the value of $\alpha=1.11 \times 10^{-2} \mathrm{Mm}^{-1}$, which was sufficiently small to reduce the periodicity effects inherent to Alissandrakis method and to provide a more robust fit with coronal loops observed by TRACE.

Figure 10 presents different views of the magnetic configuration of the active region at 07:53 UT. We can see that the active region had a multipolar magnetic configuration with six main magnetic flux systems: two low-lying central arcades (indicated by red and magenta lines and characterized by maximum heights of $\sim 42600$ and $\sim 25600 \mathrm{~km}$ respectively) superimposed on the neutral lines $L_{C}$ and $L_{A}-L_{B}$; two smaller arcades (blue and yellow lines, at heights of $\sim 4100 \mathrm{~km}$ and $\sim 2700 \mathrm{~km}$ respectively, indicated by an arrow in Fig. 10c), connecting the negative intrusion with the northern and the southern positive polarities and overhanging parts of the neutral lines $L_{C}$ and $L_{A}-L_{B}$; and two higher arcades (green lines, with a height of $\sim 125000 \mathrm{~km}$ ), the southern overhanging the arcades containing the neutral lines $L_{A}-L_{B}$, and the northern overhanging the small arcades in the negative intrusion.

In particular, Fig. 10a reports an eye-bird view of the forcefree field extrapolation; in Fig. 10b, the field of view has been rotated counterclockwise by $\sim 30^{\circ}$ to be able to indicate the field line inclination; in Fig. 10c, the field of view is rotated counterclockwise by $\sim 90^{\circ}$ and has an inclination of $\sim 60^{\circ}$ to highlight the small arcades connecting the negative intrusion with the positive polarities (indicated by an arrow); Fig. 10d reports a side view showing that the higher field lines (green lines) overlay the magenta arcade bridging the neutral lines $L_{A}-L_{B}$ and the blue yellow arcades overhanging part of $L_{C}$.

In Fig. 11, we report the force-free field extrapolations on: (a) the WL image; (b) the $\mathrm{H}_{\alpha}$ image; (c) the $1600 \AA$ image, and (d) the MDI magnetogram with RHESSI hard X-ray isocontours.

To highlight the presence of null points in the magnetic configuration, we applied a code that allows us, after the full three-dimensional magnetic field distribution has been calculated, to determine the location of these points. The code attempts to detect any change in the direction of the magnetic field vectors surrounding each point. When significant changes in the direction of the magnetic field are found, namely, a minimum in the dot product of the field vectors at two adjacent positions, the next step is to search for other minima both nearby and along field lines passing close to that point (Ugarte-Urra et al. 2007).

The results obtained by this method are shown in Fig. 12, where we can see that in the active region there are two null points relevant to the eruption: the former (shown in Fig. 12), referred to as null point 1 , is located at a height of $\sim 2300 \mathrm{~km}$ in the area of the negative polarity intrusion, which is where the small magnetic arcades are located, while the null point 2 is located on the east of filament $\mathrm{C}$, at a height of $\sim 1000 \mathrm{~km}$.

Similar results, concerning the presence of a null point with reversed Y-shape, were obtained by Régnier et al. (2004) in the non-linear force-free field approximation using vector magnetograms (IVM/MSO).

\section{Discussion}

To interpret the sequence of events preceding the X17.2 flare, we have analyzed the phenomena observed, referring to the magnetic configuration inferred from the force-free field extrapolation.

Before the preflare phase, the western (magenta) arcade overhanging the neutral lines $L_{A}$ and $L_{B}$ was affected by a destabilization due to the eruption of filament A (see Figs. 10 and 11b). The radio pre-flare activity (see Fig. 7) and the hard X-ray isocontours in Fig. 9a indicate that during this time the region overlaying the site in which the filaments $\mathrm{A}$ and $\mathrm{B}$ are closer, was characterized by particle acceleration, probably related with a vertical current sheet formed below the rising filament.

The eruption of filament $\mathrm{A}$ and lift-off of the magenta lines destabilizes the separatrix surface associated with the neutral points. TRACE images at $1600 \AA$ illustrate the effect of this destabilization from 08:16 UT, when the increase in brightness in the regions $\alpha, \beta, \gamma$, and $\epsilon$ is observed. The sites $\alpha, \beta, \gamma$, and $\epsilon$ in fact correspond to the footpoints of the separatrix surfaces associated with both null points (see Figs. 11c and 12). Further observational evidence of reconnection occurring in the field lines connected with the null point 1 is given by the hard X-ray isocontours reported in Figs. 9b, 11d, and 12, indicating the presence of accelerated particles at the site where this point is located. 


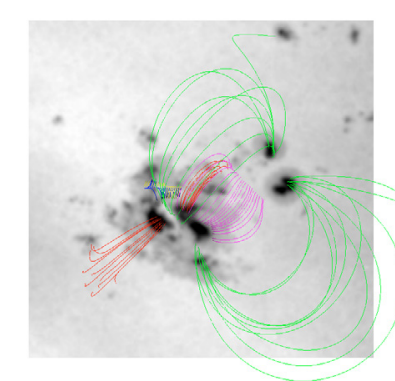

(a)

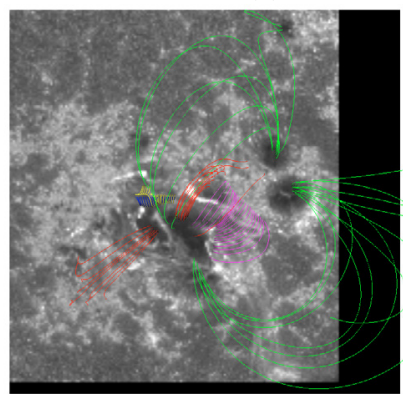

(c)

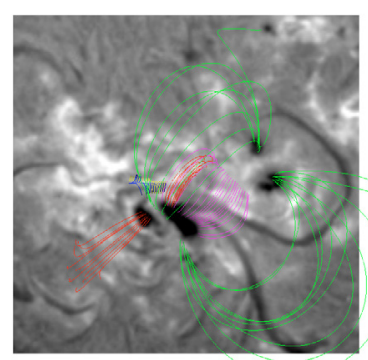

(b)

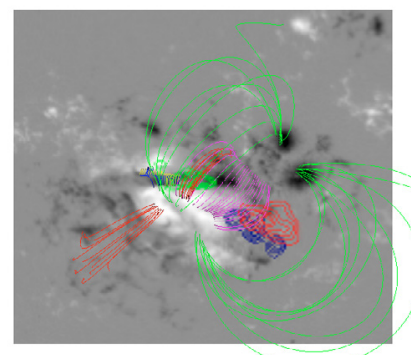

(d)

Fig. 11. Overplot of the force-free field extrapolation carried out using MDI magnetogram acquired at 07:53 UT on: a) WL image; b) $\mathrm{H}_{\alpha}$ image; c) $1600 \AA$ TRACE image; d) MDI magnetogram with RHESSI hard X-ray isocontours.

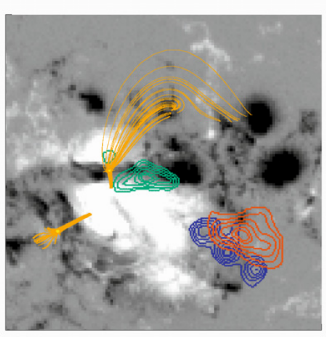

(a)

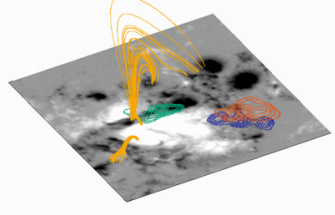

(b)

Fig. 12. Magnetic field lines and null points overplotted on the MDI magnetogram acquired at 07:53 UT: the null point 1 (see text) is located in the proximity of the negative intrusion, while the null point 2 is located on the eastern side of filament $\mathrm{C} ; \mathbf{a}$ ) and b) show two different views of the MDI magnetogram with the locations of the null points and the hard X-ray sources at 07:55:42 UT (blue), 08:16:30 UT (green), and 11:22:08 UT (red-brown).

We also emphasize the time coincidence between the occurrence of the hard X-ray emission close to null point 1 at 08:16:30 UT and the beginning of TRACE $1600 \AA$ brightenings. The position of this hard X-ray source (see Fig. 12a) indicates that it cannot be due to the vertical current sheet that formed below the rising filament $\mathrm{A}$. The presence of the hard X-ray source close to null point 1 provides additional information to previous results obtained by Mandrini et al. (2006), who found that this null point was responsible for steady EUV emission observed by TRACE, which they considered to be independent of the main flare. However, we are unable to determine for how long this hard X-ray emission continued, due to the eclipse of the RHESSI satellite at around 08:18 UT.

The regions $\alpha, \beta, \gamma$, and $\epsilon$ are not observed at $195 \AA$, probably because the energy supplied by the reconnection does not accelerate particles so as to provide observable effects at these wavelengths. Nevertheless, some signatures are observed at radio wavelengths, where successive pulses appear during this phase. More specifically, the presence of high-frequency type III bursts (1-2 GHz) during the time interval 08:00-10:15 UT, implies that reconnection occurs close to the transition region.

The reconnection at neutral point 1 continues until the $\mathrm{C}$ arcade is heated by particles accelerated at the reconnection site. In fact, TRACE observations at $195 \AA$ acquired before the beginning of the impulsive phase of the flare (between 09:08 and 09:53 UT and around 10:05 UT) exhibit a brightness increase followed by a gradual weakening of the region $\beta^{\prime}$, which corresponds to the eastern footpoint of the separatrix surface associated with the neutral point 1 (compare Fig. 5a with Fig. 12).

The lift-off of the magenta lines and the consequent decrease in tension inside the central, higher, arcade destabilize filament B; this is evident in TRACE images, pertaining to both the preflare and impulsive phases, as successive "lengthening" of region $\beta$, interspersed with gradual weakening, which finally lead to the eruption of filament B. During this eruption, X-ray emission was recorded by GOES (flare impulsive phase). As 
reconnection continued, the reconnected field lines under filament B formed post-flare loops in emission, while ribbons $R_{1}$ and $R_{2}$, in correspondence with their footpoints were observed in TRACE images at $195 \AA$ (Fig. 5).

As the number of reconnected field lines increased, the reconnection point rose, causing an increasing in the height of the post-flare loops and the separation of the ribbons with an initially high speed that subsequently decreased. This interpretation is further confirmed by the hard X-ray isocontours on TRACE images shown in Figs. 9c and d, indicating that in this phase the sites at which accelerated particles hit the lower atmosphere are confined to the B arcade. The tendency of the post-flare loops to align along the direction normal to the solar surface and perpendicularly to the neutral line, as inferred from the inclination and azimuthal angles, demonstrates that the reconnected magnetic field lines adopt a lower energy configuration. This agrees with the theory describing the relaxation of the magnetic field after eruptions, and with the result obtained by Wang et al. (2004), who found that, immediately after the X17.2 flare, some penumbral segments decayed rapidly and permanently, indicative of a more vertical magnetic field.

\section{Conclusions}

We have studied the main characteristics of the event under study, such as:

1) Multipolar magnetic field configuration.

2) Activation and/or eruption of three filaments.

3) Brightenings during the preflare and impulsive phases in sites corresponding to separatrix surfaces;

4) Post-flare loops observed almost simultaneously in distant arcades.

We believe that these characteristics could be interpreted in terms of successive destabilizations of the magnetic field configuration, by a domino effect: filament $\mathrm{A}$ eruption $\rightarrow$ lift-off of the inner (magenta) arcade $\rightarrow$ reconnection at null points located in the lower atmosphere $\rightarrow$ decrease of tension in the higher (green) arcade $\rightarrow$ destabilization of filaments B and C $\rightarrow$ X17.2 flare.

We emphasize that, even if in previous papers this event had been interpreted in terms of the breakout model (Mandrini et al. 2006; Schmieder et al. 2006a,b), in our analysis we found no evidence of the quadrupolar magnetic topology above the erupting filament required by this model, nor the expected EUV emission at the footpoints of the higher (green) loops (see Aulanier et al. 2000 and Ugarte-Urra et al. 2007).

We also emphasize that previous analyses did not take account of all the phenomena that occurred before the X17.2 flare, i.e. the eruption of filament $\mathrm{A}$, the discontinuous and intermittent brightening of structures located between the first erupting filament and the arcades supporting the other filaments, the highfrequency bursts indicative of reconnection processes during the long pre-flare phase, and the sudden and impulsive brightening of the $\mathrm{C}$ arcade that occurred almost simultaneously with that occurred in the B arcade.

We therefore propose a sounder interpretation that takes into account the initial eruption of filament A, previously neglected; this eruption would trigger a sort of domino effect, a sequence of phenomena ultimately causing the reduction in the magnetic tension of the higher arcades such that consequently both filaments $\mathrm{B}$ and $\mathrm{C}$ were free to erupt.

Acknowledgements. The authors wish to thank the referee for her/his comments and suggestions, which led to a sounder version of the manuscript. Thanks are also due to E. Catinoto and P. Costa for their cooperation in acquiring WL and $\mathrm{H} \alpha$ images at the INAF-Catania Astrophysical Observatory, to L. Vlahos for providing the code used to extrapolate the force-free magnetic field and to Dr. H. Aurass for the $40-800 \mathrm{MHz}$ Potsdam-Trensdorf spectra. This work was supported by the Istituto Nazionale di Astrofisica (INAF), by the Catania University, by the Agenzia Spaziale Italiana (contract I/035/05/0) and by the European Commission through the SOLAIRE Network (MRTN-CT-2006-035484). This research was also supported by Grant IAA300030701 of the Grant Agency of the Academy of Sciences of the Czech Republic.

\section{References}

Aulanier, G., DeLuca, E. E., Antiochos, S. K., McMullen, R. A., \& Golub, L. 2000, ApJ, 540, 1126

Alissandrakis, C. E. 1981, A\&A, 100, 197

Antiochos, S. K., DeVore, C. R., \& Klimchuk, J. A. 1999, ApJ, 510, 485

Aurass, H., Mann, G., Rausche, G.,\& Warmuth, A. 2006, A\&A, 457, 681

Aurass, H., Rausche, G., \& Mann, G. 2007, A\&A, 471, L37

Berger, T. E., \& Lites, B. W. 2003, Sol. Phys., 213, 213

Carmichael, H. 1964, in Proc. of the AAS-NASA Symposium - The Physics of Solar Flares, ed. N. Wilmot Hess, Washington, National Aeronautics and Space Administration, Science and Technical Information Division, 451 Chae, J. 2001, ApJ, 560, L95

Contarino, L., Romano, P., \& Zuccarello, F. 2006, Mem. S.A. It. Suppl., 9, 94 Hirayama, T. 1974, Sol. Phys., 34, 323

Hood, A. W., \& Priest, E. R. 1981 Geophys. Astrophys. Fluid Dyn. 17, 29

Howard, R. F., Harvey, J. W., \& Forgach, S. 1990, Sol. Phys., 130, 295

Hurford, G. J., Krucker, S., Lin, R. P., et al. 2006, Sol. Phys., 644, L93

Jiřička, K., Karlický, M., Kepka, O., \& Tlamicha, A. 1993, Sol. Phys., 147, 203

Karlický, M. 2004, A\&A, 417, 325

Kliem, B., Karlický, M., \& Benz, A. O. 2000, A\&A, 360, 715

Kopp, R. A., \& Pneuman, G. W. 1976, Sol. Phys., 50, 85

Li, H., Schmieder, B., Aulanier, G., \& Berlicki, A. 2006, Sol. Phys., 237, 85

Li, C., Tang, Y. H., Dai, Y., Fang, C., \& Vial, J.-C. 2007, A\&A, 472, 283

Lin, R. P., Dennis, B. R., Hurford, G. Y., et al. 2002, Sol. Phys., 210, 83

Loughhead, R. E., Wang, J. L., \& Blows, G. 1983, ApJ, 274, 883

Mandrini, C. H., Démoulin, P., Schmieder, B., et al. 2006, Sol. Phys., 238, 293

Martin, S. F. 2003, Adv. Space Res., 32(10), 1883

Ohyama, M., \& Shibata, K. 1998, ApJ, 499, 934

Pick, M., Malherbe, J.-M., \& Kerdraon, A. 2005, ApJ, 631, L97

Priest, E., \& Forbes, T. 2000, Magnetic Reconnection (Cambridge: Cambridge University Press)

Rausche, G., Aurass, H., Mann, G., Karlický, M., \& Vocks, C. 2007, Sol. Phys. 245,327

Régnier, S., \& Fleck, B. 2004, in Proc. of the SOHO 15 Workshop - Coronal Heating, ed. R. W. Walsh, J. Ireland, D. Danesy, \& B. Fleck, ESA Publications Division, Noordwijk, ESA SP-575, 519

Roumeliotis, G., Sturrock, P. A., \& Antiochos, S. K. 1994, ApJ, 423, 847

Rust, D. M. 2003, Adv. Space Res., 32(10), 1895

Schmieder, B., Mandrini, C. H., Démoulin, P., et al. 2006a, Adv. Space Res., 37, 1313

Schmieder, B., Mandrini, C. H., Berlicki, A., Démoulin, P., \& Li, H. 2006b, in Proc. of the SOHO-17 Conf. - 10 Years of SOHO and Beyond, ed. H. Lacoste, \& L. Ouwehand, European Space Agency, ESA SP-617, 145

Sturrock, P. A. 1966, Nature, 211, 695

Su, Y. N., Golub, L., van Ballegooijen, A. A., \& Gros, M. 2006, Sol. Phys., 236, 325

Török, T., \& Kliem, B. 2005, ApJ, 630, L97

Trottet, G., Krucker, S., Lüthi, T., \& Magun, A. 2008, ApJ, in press

Ugarte-Urra, I., Warren, H. P., \& Winebarger, A. R. 2007, ApJ, 662, 1293

Wang, H., Liu, C., Qiu, J., et al. 2004, ApJ, 601, L195

Williams, D. R., Török, T., Démoulin, P., van Driel Gesztelyi, L., \& Kliem, B. 2005, ApJ, 628, L163 